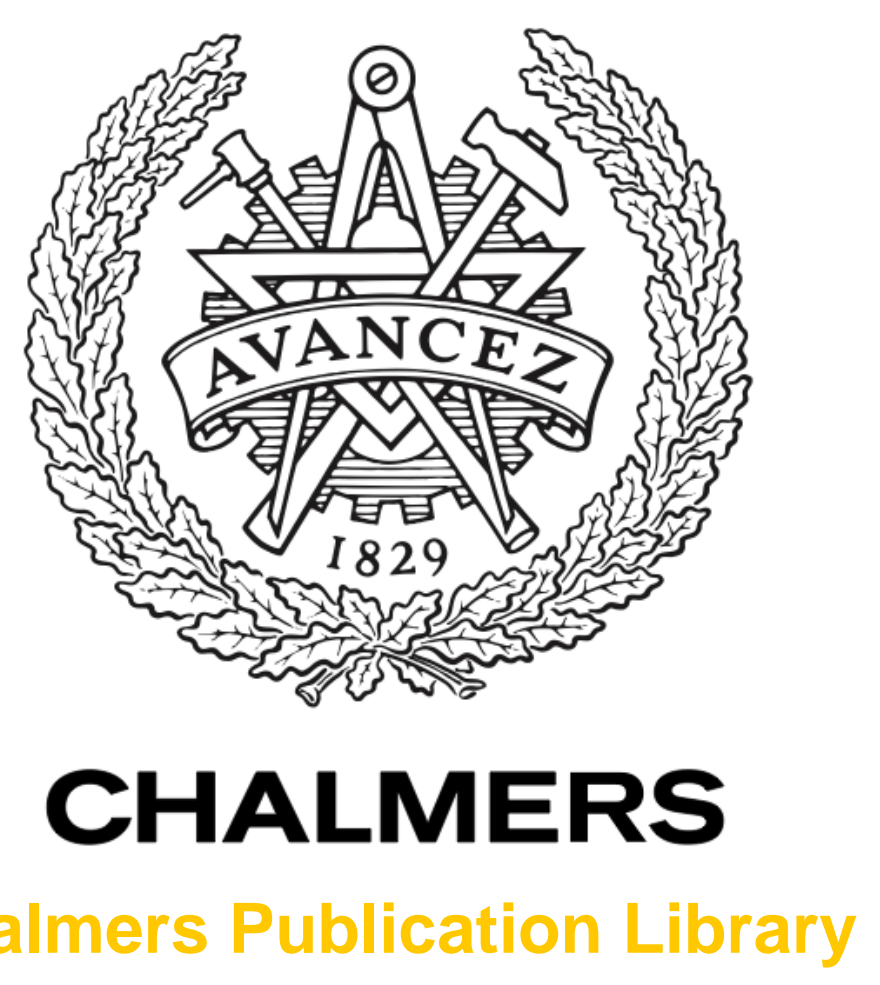

Chalmers Publication Library

\author{
Biorthogonal modulation in 8 dimensions experimentally implemented as 2PPM-PS- \\ QPSK
}

This document has been downloaded from Chalmers Publication Library (CPL). It is the author's version of a work that was accepted for publication in:

Technical Digest Series: Optical Fiber Communication Conference, OFC 2014; San Francisco, CA; United States; 9 March 2014 through 13 March 2014 (ISSN: 2162-2701)

Citation for the published paper:

Eriksson, T. ; Johannisson, P. ; Agrell, E. (2014) "Biorthogonal modulation in 8 dimensions experimentally implemented as 2PPM-PS-QPSK". Technical Digest Series: Optical Fiber Communication Conference, OFC 2014; San Francisco, CA; United States; 9 March 2014 through 13 March 2014 pp. Art. no. 6886984.

http://dx.doi.org/10.1109/OFC.2014.6886984

Downloaded from: http://publications.lib.chalmers.se/publication/197743

Notice: Changes introduced as a result of publishing processes such as copy-editing and formatting may not be reflected in this document. For a definitive version of this work, please refer to the published source. Please note that access to the published version might require a subscription.

Chalmers Publication Library (CPL) offers the possibility of retrieving research publications produced at Chalmers University of Technology. It covers all types of publications: articles, dissertations, licentiate theses, masters theses, conference papers, reports etc. Since 2006 it is the official tool for Chalmers official publication statistics. To ensure that Chalmers research results are disseminated as widely as possible, an Open Access Policy has been adopted.

The CPL service is administrated and maintained by Chalmers Library. 


\title{
Biorthogonal Modulation in 8 Dimensions Experimentally Implemented as 2PPM-PS-QPSK
}

\author{
Tobias A. Eriksson ${ }^{1}$, Pontus Johannisson ${ }^{1}$, Erik Agrell ${ }^{2}$, Peter A. Andrekson ${ }^{1}$, Magnus Karlsson ${ }^{1}$ \\ ${ }^{1}$ Photonics Laboratory, Department of Microtechnology and Nanoscience. ${ }^{2}$ Department of Signals and Systems. \\ Chalmers University of Technology, SE-412 96 Göteborg, Sweden, tobias.eriksson@chalmers.se
}

\begin{abstract}
We experimentally demonstrate biorthogonal modulation in 8 dimensions as binary pulse-position modulation polarization-switched QPSK. We compare this format with PM-QPSK at the same bit rate and show a $1.4 \mathrm{~dB}$ sensitivity gain and $84 \%$ increased transmission distance.

OCIS codes: (060.1660) Coherent communications; (060.4080) Modulation; (060.2330) Fiber optics communications
\end{abstract}

\section{Introduction}

4-dimensional (4D) modulation formats were introduced in 2009 by Bülow [1] as well as Karlsson and Agrell [2] and it initiated a research trend on modulation formats that are optimized in the 4D signal space spanned by the two quadratures and the two polarization states of the optical field. Polarization-switched quadrature phase-shift keying (PS-QPSK) was found to be the 4D modulation format with the highest asymptotic power efficiency (APE), having a $1.76 \mathrm{~dB}$ better APE than polarization multiplexed (PM) QPSK [2]. It has been verified in experiments that the higher APE can be translated into an increase in transmission reach over PM-QPSK [3-5]. Another format with the same APE as PS-QPSK is binary pulse-position modulation QPSK (2PPM-QPSK), which has been shown to achieve a similar increase in reach over PM-QPSK in long-haul transmission links [6]. Other 4D modulation formats include the set-partitioning (SP) family [7], where subsets of 4D quadrature amplitude modulation (QAM) constellations with increased APE are identified using SP. For instance, by using SP on PM-16QAM the subset 128-ary SP-QAM (128-SP-QAM) with $2.43 \mathrm{~dB}$ increased APE over PM-16QAM is found. 128-SP-QAM has been shown to achieve longer transmission distances compared to PM-16QAM in experiments $[8,9]$. The higher APE for both PS-QPSK and the SP-family comes at the cost of a reduced spectral efficiency (SE). These 4D formats, including polarization QAM modulation (POL-QAM) [1], have been suggested for flexible networks since they can fill the gap in terms of transmission reach and bit-rate between QAM-constellations of different sizes [10].

The 4D modulation space is realized by the two quadratures and the two polarization states of the optical field. 8-dimensional (8D) modulation formats were recently investigated for coherent optical systems where the dimensionality can be increased to 8 by using two frequencies [11] or two consecutive time slots [12]. Due to the increased degrees of freedom, modulation formats that are optimized in the 8D signal space has the potential to achieve higher APE and/or higher SE than the 4D formats. In [12], 8D modulation formats with sensitivities comparable to PS-QPSK but with higher SE are proposed and numerically investigated. Biorthogonal modulation in 8 dimensions was experimentally investigated in [11] as 2-ary frequency shift keying combined with PS-QPSK, the format was denoted 4-ary frequency and polarization switched QPSK. This format was compared against dual-carrier (DC) PM-QPSK (DC-PMQPSK) and DC-PS-QPSK at the same symbol rate and DC were used to compare the formats with the same spectral width. It was found that the transmission distance could be increased by $84 \%$ over DC-PM-QPSK [11]. Compared to the proposed formats in [12], biorthogonal modulation in 8 dimensions can achieve the highest APE and it can be implemented using binary driving signals. However, the SE is lower than the $8 \mathrm{D}$ formats in [12].

In this paper we experimentally demonstrate biorthogonal modulation in 8 dimensions using two consecutive time slots to achieve the 8 dimensions. The format is implemented as 2PPM in combination with PS-QPSK and we denote this format 2PPM-PS-QPSK. To our knowledge, this is the first experimental demonstration of 2PPM-PS-QPSK. We compare this format to PM-QPSK in back-to-back and long-haul experiments at the same bit rate of $85.6 \mathrm{Gbit} / \mathrm{s}$. We demonstrate an increased sensitivity of $1.4 \mathrm{~dB}$ for 2PPM-PS-QPSK over PM-QPSK at a bit-error rate (BER) of $10^{-3}$ and show transmission of 2PPM-PS-QPSK of up to $12,300 \mathrm{~km}$ which is an increase of $84 \%$.

\section{2PPM-PS-QPSK}

The symbols of $8 \mathrm{D}$ biorthogonal modulation has 16 levels which can be described by all permutations of $\left\{ \pm 1,0^{7}\right\}$ [13]. We realize this format by transmitting one QPSK symbol in one of the four positions given by two time slots and two 


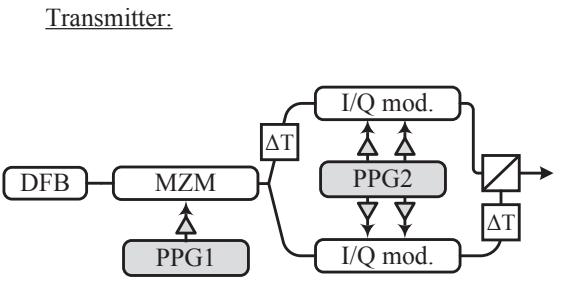

(a)

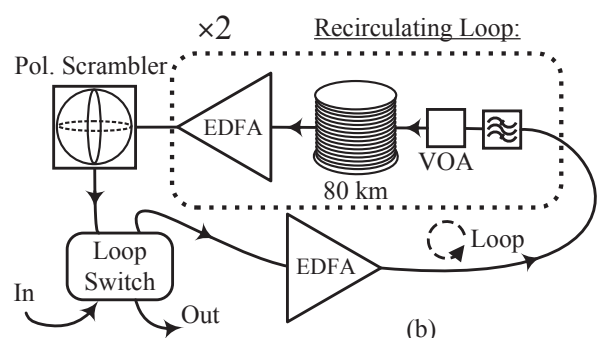

(b)

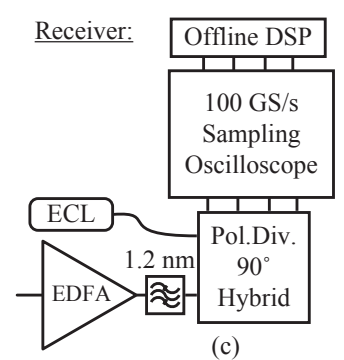

(c)

Fig. 1. Experimental setup showing: (a) The transmitter for both PM-QPSK and 2PPM-PS-QPSK. When PM-QPSK is modulated the driving signal to the MZM is disabled. (b) The recirculating loop consisting of two spans of $80 \mathrm{~km} \mathrm{SMF.} \mathrm{(c)} \mathrm{The} \mathrm{coherent} \mathrm{receiver} \mathrm{setup.}$

polarizations. This can be seen as combining 2PPM with PS-QPSK, where two bits are encoded in the QPSK symbol and one bit each in the selection of time slot and polarization.

The APE for a modulation format with minimum Euclidean distance $d_{\min }$ is defined as $\gamma=d_{\min }^{2} \log _{2} M /\left(4 E_{s}\right)$ where $M$ is the number of symbols and $E_{s}$ is the average energy per symbol [2]. The factor of $1 / 4$ is used to normalize the APE to QPSK. In combination with the APE the SE is often used and it is defined as $S E=\log _{2}(M) /(N / 2)$ where $N$ is the dimensionality of the format [2]. PM-QPSK has APE $=0 \mathrm{~dB}$ and $\mathrm{SE}=2 \mathrm{bit} / \mathrm{symbol} /$ polarization and 2PPM$\mathrm{PS}-\mathrm{QPSK}$ has $\mathrm{APE}=3 \mathrm{~dB}$ and $\mathrm{SE}=1 \mathrm{bit} / \mathrm{symbol} /$ polarization. To our knowledge, this is the most power efficient modulation format in 8 dimensions described to date.

\section{Experimental Setup}

The experimental setup is shown in Fig. 1. The transmitter for 2PPM-PS-QPSK is based on first generating 2PPM using a Mach-Zehnder Modulator (MZM) driven with a 2PPM pattern, generated from pseudo-random binary sequence (PRBS) data with the length $2^{15}$, with a pulse repetition rate of $42.8 \mathrm{GHz}$ generated with a pulse-pattern generator (PPG). The PPM-pulses are then modulated with PS-QPSK with the use of two IQ-modulators, one for each polarization, driven at a symbol rate of 21.4 Gbaud. Three of the input signals to the IQ-modulators are PRBS sequenecs with the length of $2^{15}-1$ and the fourth driving signal is formed by an XOR-operation on the other three to generate PS-QPSK. As light source, a distributed feedback laser (DFB) with $\sim 100 \mathrm{kHz}$ linewidth is used and optical delay-lines in combination with electrical delays are used to synchronize the different modulators. When PM-QPSK is generated, the driving signal to the MZM is simply switched off and a PRBS sequence is used instead of the XOR-sequence.

The signals are propagated in a recirculating loop, shown in Fig. 1(b). The loop consists of two spans of $80 \mathrm{~km}$ single mode fiber (SMF) which are preceded by variable optical attenuators (VOA) to control the launch power into each span and optical bandpass-filters with $4 \mathrm{~nm}$ bandwidth, to suppress out-of-band amplified spontaneous emission (ASE) noise. The two spans are followed by erbium doped fiber amplifiers (EDFA) with $\sim 5 \mathrm{~dB}$ noise figures to compensate for the span-loss and a third EDFA is used to compensate for the loss of the loop-switching components and the polarization scrambler. The polarization scrambler prevents any unrealistic accumulation of polarization effects and is synchronized to the loop roundtrip time.

The coherent receiver, shown in Fig. 1(c), consists of a polarization diverse $90^{\circ}$ optical hybrid with integrated balanced detectors. $\mathrm{A} \sim 300 \mathrm{kHz}$ linewidth external cavity laser (ECL) is used as a local oscillator (LO). The electrical signals from the hybrid are sampled using a $100 \mathrm{GS} / \mathrm{s}$ real-time sampling oscilloscope with $33 \mathrm{GHz}$ bandwidth. The signals are then processed off-line. The digital signal processing (DSP) is almost identical for the two formats. Impairments from the optical front-end are compensated followed by re-sampling to 2 samples/symbol and chromatic dispersion compensation. After that, polarization demultiplexing and adaptive equalization is performed using the constant modulus algorithm (CMA) for PM-QPSK and a modified version of the PS-CMA [14] with a power threshold to determine which PPM-slot that was transmitted. The equalizer taps are not updated for the samples with power lower than the threshold. Next, a frequency offset estimation based on the fast-Fourier transform and a block-wise phase estimation based on the Viterbi-Viterbi algorithm with a block-size of 128 samples for 2PPM-PS-QPSK and 64 samples for PM-QPSK are performed. Finally, the BER is evaluated.

\section{Results}

The back-to-back experimental results as well as the analytical predictions for PM-QPSK and 2PPM-PS-QPSK at 85.6 Gbit/s are shown in Fig. 2(a) as the BER as a function of OSNR $(0.1 \mathrm{~nm})$. At a BER of $10^{-3}$, indicated with 

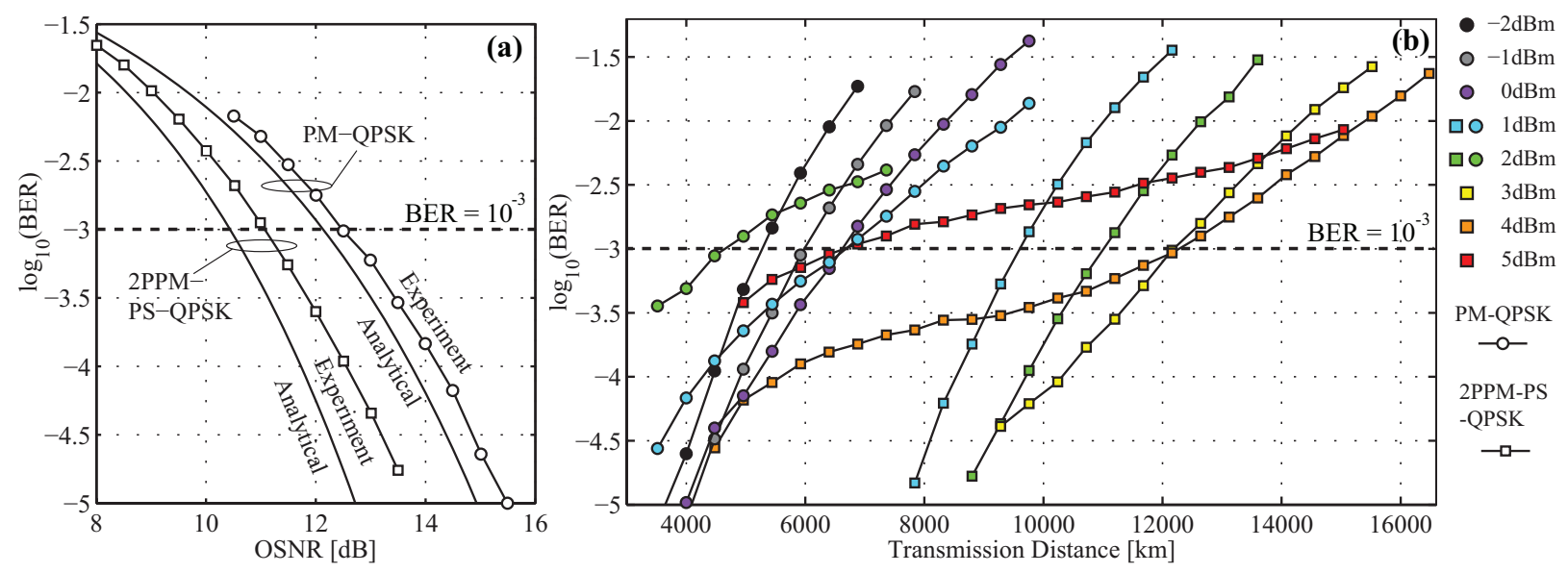

Fig. 2. (a) Measured back-to-back BER as a function of OSNR for $85.6 \mathrm{Gbit} / \mathrm{s}$ PM-QPSK (circles) and 85.6 Gbit/s 2PPM-PS-QPSK (squares). Analytical predictions are shown as solid lines. (b) BER as a function of transmission distance for different launch powers into the $80 \mathrm{~km}$ SMF spans for $85.6 \mathrm{Gbit} / \mathrm{s}$ PM-QPSK (circles) and $85.6 \mathrm{Gbit} / \mathrm{s}$ 2PPM-PS-QPSK (squares). The colors mark the launch power. The optimal launch power at $\mathrm{BER}=10^{-3}$ (dashed line) is $1 \mathrm{dBm}$ (blue) for PM-QPSK and $4 \mathrm{dBm}$ (orange) for 2PPM-PS-QPSK.

dashed horizontal lines in Fig. 2, the implementation penalty is $0.3 \mathrm{~dB}$ for PM-QPSK and $0.6 \mathrm{~dB}$ for 2PPM-PSQPSK. This gives an experimental sensitivity gain of $1.4 \mathrm{~dB}$ compared to expected gain of $1.7 \mathrm{~dB}$ from the analytical predictions and the expected $3 \mathrm{~dB}$ asymptotic sensitivity gain. As seen from the analytical plots, the sensitivity gain is decreasing with OSNR.

The BER as a function of transmission distance for different launch powers for the two formats are shown in Fig. 2(b). We define the transmission reach as the distance where the BER $=10^{-3}$. PM-QPSK can be transmitted up to $6,700 \mathrm{~km}$ at the optimal launch power of $1 \mathrm{dBm}$ and 2PPM-PS-QPSK up to $12,300 \mathrm{~km}$ at the optimal launch power of $4 \mathrm{dBm}$. This corresponds to an increase in transmission reach of $84 \%$ when the two formats are compared at the same bitrate of $85.6 \mathrm{Gbit} / \mathrm{s}$. It should be noted that since the number of bits per $8 \mathrm{D}$-symbol of 2PPM-PS-QPSK is half compared to PM-QPSK over two bit slots, the pulse repetition rate (which well approximates the occupied bandwidth) is doubled, $41.8 \mathrm{GHz}$ compared to $21.4 \mathrm{GHz}$, to achieve the same bit rate. This means that the spectrum is much broader for 2PPM-PS-QPSK which could explain why we see significant penalty from non-linear effects at shorter transmission reaches and high launch power for 2PPM-PS-QPSK.

At long transmission reaches where the BER is much higher than $10^{-3}$ and approaches 0.1 , both formats suffers from cycle slips in the phase estimation. We also notice that the convergence of the modified version of the PSCMA [14] was sensitive to how the power threshold was selected. However, when the threshold was optimized, the misconvergence rate was less than $5 \%$ for any transmission distance. This problem, and ambiguities, could be solved by the use of training sequences.

\section{Conclusions}

We have demonstrated a possible implementation of $8 \mathrm{D}$ biorthogonal modulation as 2PPM-PS-QPSK using two consecutive time-slots to achieve 8 dimensions. We compared this format to PM-QPSK at the same bitrate of $85.6 \mathrm{Gbit} / \mathrm{s}$ and show an $1.4 \mathrm{~dB}$ back-to-back sensitivity gain over PM-QPSK. We have shown transmission of up to $12,300 \mathrm{~km}$ which corresponds to an increase of $84 \%$ over PM-QPSK.

\section{References}

1. H. Bülow, OFC'09, Paper OWG2, 2009.

2. E. Agrell and M. Karlsson, J. Lightwave Technol., vol. 27, no. 22, pp. 5115-5126, 2009.

3. M. Sjödin, et al., Opt. Exp., vol. 19, no. 8, pp. 7839-7846, 2011.

4. J. K. Fischer, et al., Opt. Exp., vol. 19, no. 19, pp. B667-B672, 2011.

5. M. Nölle, et al., Opt. Exp., vol. 19, no. 24, pp. 24370-24375.

6. M. Sjödin, et al., $O F C^{\prime} 13$, Paper OTu2B.7, 2013.

7. L. Coelho and N. Hanik, OFC'11, Paper Mo.2.B.4, 2011.

8. T. A. Eriksson, et al., Opt. Exp., vol. 21, no. 16, pp. 19269-19279, 2013.

9. J. Renaudier, et al., OFC'13, Paper OTu3B.1, 2013.

10. J. K. Fischer, et al., ECOC'13, Paper Tu.3.C.1, 2013.

11. T. A. Eriksson, et al., ECOC'13, Paper Th.2.D.4, 2013.

12. T. Koike-Akino, et al., ECOC'13, Paper Tu.3.C.3, 2013.

13. M. K. Simon et al., Digital Communication Techniques: Signal Design and Detection, Prentice Hall, 1995.

14. P. Johannisson et al., Opt. Exp., vol. 19, no. 8, pp. 7734-7741, 2011 